\title{
DELIO TESSA STUDENTE ALL'UNIVERSITÀ DI PAVIA
}

\author{
Nota del m.e. MARIO PISANI (*)
}

(Adunanza dell'8 maggio 2014)

SuNTO. - Delio Tessa (1886-1939), scrittore e poeta dialettale milanese, ha compiuto gli studi universitari a Pavia, frequentando la Facoltà di Giurisprudenza. La sua carriera di studente non fu particolarmente brillante, e, come risulta dai suoi scritti, pubblicati dopo la morte, il ricordo di quella città rimase solo di tipo sentimentale e fonte di ispirazione poetica.

$$
* * *
$$

ABSTRACT. - Delio Tessa (1886-1939), the writer and dialect poet of Milan has accomplished his studies at the University of Pavia, attending the Faculty of Law. His career as a student was not particularly brilliant and, as it results from his prose writings, published after his death, his recollections of that town remained only sentimental and a source of poetic inspiration.

1. Delio Tessa nasce a Milano il 18 novembre 1886, e dopo aver, piuttosto tardivamente (a vent'anni) conseguito il diploma di licenza liceale presso il Regio Liceo Cesare Beccaria di questa città, si iscrive alla Facoltà di Giurisprudenza dell'Università di Pavia per l'anno accademico 1906-1907.

All'epoca, e lo sarà ancora per ben oltre un decennio, quella pavese

(*) Università degli Studi di Milano, Italia. E-mail: mariopisani.mp@libero.it 
era l'unica Università della Lombardia, e nella Facoltà giuridica tenevano cattedra personalità scientifiche di prima grandezza, a livello nazionale: tra questi, Oreste Ranelletti, per Diritto amministrativo e Scienza dell'amministrazione; Pasquale Del Giudice, per Storia del diritto italiano; Pietro Bonfante, per Diritto romano; Ercole Vidari, per Diritto commerciale; Giulio Cesare Buzzati (il padre di Dino) per Diritto internazionale.

Consultando gli scrittori che hanno avuto modo di occuparsi della biografia del nostro poeta, non era (e non è) raro imbattersi in espressioni, a lui riferite, di questo genere: "studi assai poco brillanti"; nel 1911 conquista "una stentata laurea in giurisprudenza"; "con fatica e nessun entusiasmo".

Sarà anche - anticipiamolo - che il peso degli anni può acuire una particolare sensibilità, e magari, o appena soltanto, può accentuare gli spigoli di una qualche deformazione professionale. Fatto sta che, al postumo collega studentesco di Delio Tessa, che di lui, in remota età giovanile aveva così tanto apprezzato, affacciati su qualche antologia - intitolata, poniamo, Lirica del Novecento, del $1953^{1}$ - poesie come "I cà" (diceva: “... in qui casoni / brutt ch'hin vegnu / su come i fong / in via Bligny"), o come, ed ancor più, quell'altra, intitolata "Ciana” (“... pensi alla Ciana / incoeu ... e me domandi ...”); appunto a quel postumo collega, risolutamente poi divenuto giurista tutto d'un pezzo, era purtuttavia spiaciuto, e ancora spiaceva, veder riportate, a stampa, quelle espressioni non lusinghiere riferite ai corsi (e trascorsi) di studio del poeta vernacolare d'antan.

Certo: erano lentamente affiorati, tra le prose edite dello stesso Tessa, anche alcuni passaggi che suonavano come esplicite sue ammissioni di remote debolezze, o trascuratezze, di stampo scolastico-accademico. ${ }^{2} \mathrm{Ma}$ quelle ammissioni potevano anche essere - era dato di pensare - fluorescenze paradossali o vezzi espressivi di trasgressione umorale ed indefinita.

1 L'avevano curata, per Vallecchi, Luciano Anceschi e Sergio Antonielli.

2 In Madonnina e campanile, uno scritto dell'11 novembre 1938, pubblicato sul "Corriere del Ticino", Tessa ricorderà (ancora!) che "nella sezione [sessione] di luglio" del 1909, egli non aveva sostenuto "neanche un esame" (stava al $2^{\circ}$ anno), ma che "in compenso avev(a) aiutato a metter su la Stivaliade revue, «ferie» studentesca che tenne il cartellone per una ventina di sere tra Filo e Lirico". Ed in linea assai più "generale", l'anno prima, parlando, su "L'Ambrosiano" del 29 marzo 1937, de Il legatore di libri, aveva scritto: "... per me i libri più melanconici che mi conosco sono i fascicoli di Giurisprudenza e i trattati di diritto ... libri d'uggia!”. 
Per superare i dubbi, o la loro rimozione sentimentale, il passo che restava da compiere era però assai breve: quello, bastava solo decidersi, che portava ad accedere all'Archivio storico dell'Università di Pavia, di recente costituzione.

2. Ci è voluto poco per ritrovarci tra le mani il volumone del "Registro della carriera scolastica", e per leggervi, all'altezza di un certo n. 2929, il nome di "Tessa Delio, figlio di Senio e Clara Besozzi, nato ecc.", che "fece gli studi nel Liceo Beccaria di Milano".

Al di sotto ecco dispiegarsi due ampie facciate, dove sinotticamente si indicano, anno per anno, dal 1906 al 1910, i corsi degli insegnamenti "obbligatori", e di quelli "liberi", coi nomi dei rispettivi professori e le votazioni riportate dal candidato in ciascun esame.

Non possono che colpire, a prima lettura, la teoria dei voti, per lo più non superiori alla sufficienza, e cioè al 18 , ed anche alcune riprovazioni, espressamente verbalizzate: un 15 in Diritto commerciale, poi rimediato con un 21; un 12 in Diritto amministrativo, rimediato con un 22; un altro 15 in Procedura civile ed Ordinamento giudiziario, rimediato con un altro 21.

La media delle votazioni d'esame con la quale il candidato Tessa si presenterà alla sessione di laurea - era l'8 dicembre 1911 - era pari a punti 19; e dopo "la disputa (sic) prescritta sulla dissertazione e sulle tesi" (orali) - il tutto alla stregua dell'allora recente regolamento generale universitario 9 agosto 1910 - la votazione finale e complessiva era risultata, e non era certo un gran voto, di punti 77/110.

Per amor di verità va anche detto che, scorrendo lo stesso "Registro", risultano anche, e non proprio pochissime, defezioni studentesche nell'iter accademico, con dirottamenti verso sedi universitarie ritenute più agevoli; ma che risultano, per converso, così come votazioni di 30 trentesimi per i singoli esami, anche punteggi di laurea a livelli di pienezza: e così, ad esempio, 102/110 per Eucardio Momigliano, ${ }^{3}$ ed anche alcuni 110, oltre a un 110 e lode.

3 Di "Momi”, suo compagno all'epoca del Liceo Beccaria, tanti anni dopo, il 12 agosto 1939, dalla radio della Svizzera Italiana Tessa ricorderà anche, accanto alle sue benemerenze di "scrittore di professione", l'antico "altruistico spirito di colleganza", quando dava "da copiare ai compagni più impappinati le traduzioni dal greco e dal latino". 
3. Ma il documento più interessante dell'Archivio Storico è per noi quello costituito dal verbale (anzi: "processo verbale") di laurea del Tessa, dove si dà atto che il medesimo presenta "una dissertazione" dedicata all'argomento "Del diritto di voto alle donne", e due "tesi" orali (in seguito il gergo le chiamerà "tesine"), una in statistica e una in diritto civile.

Non è espressamente indicato il relatore della dissertazione-base, ma con ogni verosimiglianza si trattava del (presente e firmante) prof. Livio Minguzzi, costituzionalista attivo in Facoltà a decorrere dal 1899. ${ }^{4}$

Sulle prime, l'intitolazione, e quindi la scelta dell'argomento, desta simpatia, oltre ad un certo plauso per la lungimiranza. E' ben noto, infatti, che il diritto di voto alle donne (per la precisione: maggiorenni, e dunque - al tempo - ventunenni ed oltre) verrà concesso alla distanza di più d'un trentennio: col d. lgs. luogot. $1^{\circ}$ febbraio $1945, \mathrm{n}$. 23. Ma già a raffrenare, se non la prima (la simpatia), sicuramente il secondo (il plauso), è il dubbio che può insorgere - come possono chiedersi, oggi, quelli del mestiere - se a proporre l'argomento della tesi fosse stato il candidato, o non piuttosto il relatore. Anche un tale dubbio, però, viene sùbito e definitivamente (ma non brillantemente) superato. Basta consultare, infatti, per quanto attiene alla Facoltà di Giurisprudenza, il già citato testo del Regolamento generale universitario, per leggervi, all'art. 12 , comma $2^{\circ}$, la previsione che il tema della dissertazione scritta viene "liberamente scelto dal candidato in materia pertinente alla Facoltà". Si aggiunga poi, ed inoltre, la sorprendente ammissione dello stesso laureato, contenuta, ventisette anni dopo, in una "prosa ambrosiana" del 15 novembre 1938, dedicata al "pittore umanista", ed avvocato, Fortunato Rosti (il "più vecchio amico" del Tessa, che diverrà geloso custode dei suoi scritti inediti). Troviamo scritto, invero, che "Fatta la laurea lui [il Rosti] a Bologna, io a Pavia colla stessa tesi sul voto alle donne e che io bo copiato da lui e lui da un altro, capovolgendo le conclusioni; Rosti era favorevole, io contro". Null'altro; e seguiva, col solo distacco di una virgola, la breve e non congrua specificazione: "si ebbe nel '14 studio assieme in via Spiga verso il naviglio". (Nello stesso anno - aggiungiamo noi, con tentativo di lievità - era però incominciata ... anche la prima guerra mondiale!).

4 MUSSELLI, La Facoltà di Giurisprudenza di Pavia nel primo secolo dell'Italia unita, in Ann. di st. delle università italiane, n. 7/2003, 208. 
Del testo della dissertazione, ad ogni modo, a quanto pare nell'Archivio pavese è scomparsa ogni traccia.

4. Quanto alle "tesi" orali effettivamente svolte - il regolamento prevedeva che fosse ancora lo stesso candidato a indicarne tre, ed a svolgerne oralmente almeno due - la prima, in tema di statistica, concerneva un argomento piuttosto singolare, che si può ritenere di netta impronta tessiana: "Se si possa accogliere la critica che Leone Tolstoi fa della dottrina di Malthus".

La seconda "tesi" orale - secondo la dottrina del tempo prospettata come materia di "diritto civile" - era così intitolata: "Se le pellicole cinematografiche sono opere dell'ingegno": un tema che ai cultori, e ai memori lettori del Tessa, può perfino apparire come la prefigurazione di tutte quelle molteplici e disperse attenzioni che, nel corso degli anni a venire, il nostro autore dedicherà al cinematografo. ${ }^{5}$ Attenzioni culminate in una sceneggiatura cinematografica - titolo: Vecchia Europa - la cui "ideazione", poi tradottasi "in immagini di poesia", ${ }^{6}$ era "nata all'autore in un pomeriggio d'agosto al cader del sole davanti alla basilica di San Michele in Pavia".

5. Molti anni dopo, parlando di un'altra basilica, quella di San Lanfranco, Tessa dirà che lui, milanese a Pavia, aveva "il cuore", da

5 Paolo Mauri, curatore di una recente e corposa raccolta delle Prose ambrosiane e delle Critiche contro vento (intitolata La bella Milano, Macerata, ed. Quodlibet, 2013), intitola la sua introduzione Tessa, il poeta che andava al cinema. Nella sua nota bibliografica, l'A. non manca di ricordare, a guisa di "testo di riferimento", l' "ottima edizione" del Tessa, curata da Dante Isella, intitolata Ore di città (Torino 1988), e quanto alla seconda parte, "l'utilissima raccolta", a cura di Giuseppe Anceschi, dal titolo Critiche contro vento. Pagine «ticinesi» 1934-1939 (Lugano 1990). Anche l'amico Carlo Linati ( «El Tessa», in Settegiorni del 7 marzo 1943) stendendone un ritratto che sarà raccolto, con altri, in Il bel Guido e altri ritratti, Milano 1982, 60, ricorda che Tessa, negli ultimi anni, ebbe una passione "vivissima" per il cinema; e ad esso "dedicò studi, ricerche e fantasie, portando anche in quel campo propositi tutti suoi".

6 Così A. Stella, Il piano di Lucia - Manzoni e altre voci lombarde, Firenze 1999, 64 .

7 Come ricordava anche Isella, la sceneggiatura Vecchia Europa è uscita per la stampa, a cura di Cristina Sacchi, e con una premessa di Stella, presso l'editore Bompiani (Milano 1986, pp. 197). 
quando - era il 1906 - vi aveva iniziato gli studi universitari. ${ }^{8} \mathrm{Ma}$ a Pavia-città - viene dato di correggere - e ancor più alla sua stagione pavese giovanile, perché quanto, invece, alla città degli studi, comprensibilmente il Tessa non dedica particolari attenzioni di reminiscenza o di rimpianto. Nei suoi scritti figura solo, infatti, qualche cenno fugacissimo: una volta alle "dispense di economia" che lo studente portava con sé; un'altra volta al non nominato suo "professore di medicina legale", per il suo far uso di una certa qual espressione a proposito dei "vecchietti" ultra-ottantenni, "ma in gamba", un modello dei quali il Tessa individuava in un maestro di musica frequentatore di Toscanini.

Al di là di questo, che è pari a nulla, il nostro autore, prodigo invece di ricordi dei suoi professori di liceo, nulla dice (per più o meno consapevole rimozione) dei suoi professori dell'Università.

Del resto, in una sua auto-presentazione, datata 21 dicembre 1935, a Radio Monteceneri, il nostro autore, non certo, e neanche di sfuggita, dell'Alma Ticinensis Universitas veniva a parlare, ma di tutt'altro. E così aveva avuto modo di scrivere: “... Il mondo mi ha deluso e son pieno di amarezze. Se ripenso ai miei anni universitari non li so distinguere dagli ideali che rendevano in quei tempi fervida la mia giovinezza. Ideali di pace e di fratellanza. Ho assistito invece al loro triste tramonto".

Qualche anno più tardi, però, meditando in occasione del già ricordato ritorno a San Lanfranco, il cinquantatreenne Tessa sembra riprendere fiducia; e usando le stesse parole dirà: “... non l'armi come nei tempi remoti, ma le idee di fratellanza e di pace sian benedette da questi altari”.

Pochi mesi dopo, il 21 settembre 1939, la morte gli tendeva l'agguato inesorabile.

E muovendo da questo davvero finale evento, che mette decisamente fuori campo studenti e professori, le nostre disperse note vorrebbero essere, o ritornare, lievi: così come lieve si augura che sia la terra, quando un gesto simbolico d'addio ne fa calare una piccola zolla verso lo scomparso che ci è caro.

E allora, alla fine - e senza bisogno di appoggiarci agli avalli, che a Delio Tessa pur non erano mancati, di un Benedetto Croce e di un Pietro Pancrazi - ci par giusto riprendere in mano, per cambiare regi-

8 V. «L'illustrazione ticinese» del 28 gennaio 1939. 
stro, e sempre a titolo d'esempio, la poesia della Ciana (aprile 1935), da cui avevamo preso le mosse: Ciana “... rebuttada, casciada/su come tutti/i pover Crist" / "Ho mai vist, Ciana - aggiungeva il nostro poeta ona povera tosa/come ti". 
